\section{PATHOOLOGY \\ tor}

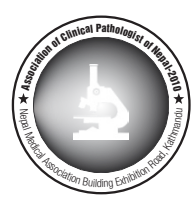

An official publication of Association of Clinical Pathologists of Nepal

Page I

ISSN:2091-0797

Septemeber 2014

Vol. 4 | No. 2 | Issue 8

\title{
Pathology Laboratories and Infection Prevention and Control
}

Laboratory health care workers are vulnerable to infection with the Hospital Acquired Infections (HAIs) while receiving, handling and disposing biological samples. Ideally the infrastructure of the lab should be according to the best practices like good ventilation, room pressure differential, lighting, space adequacy, hand hygiene facilities, personal protective equipments, biological safety cabinets etc. Disinfection of the environment, and specific precautions with sharps and microbial cultures should follow the protocols and policies of the Infection Prevention and Control Practices (IPAC). If Mycobacterium tuberculosis or Legionella pneumophila are expected, diagnostic tests should be performed in a bio-safety level 3 facilities (for agents which may cause serious or potentially lethal disease in healthy adults after inhalation). Laboratory access should be limited only to people working in it.

Along with the advent of new technologies and advanced treatment we are now facing problems with the dreadful HAIs with Antimicrobial Resistant Organisms (AROs) which is taking a pandemic form. According to WHO, hundreds of millions of patients develop HAI every year worldwide and as many as 1.4 million occur each day in hospitals alone. The principal goals for hospital IPAC programs are to protect the patient, protect the health care worker (HCW), visitors, and other persons in the health environment, and to accomplish the previous goals in a cost-effective manner like hand hygiene, surveillance, training of the HCWs, initiating awareness programs and making Best Practices and Guidelines to be followed by everyone in the hospital.

The initiation for the best practices in the Pathology Laboratories can be either Sporadic or Organizational. Sporadic initiation is when the laboratories make their own IPAC policies. It has been seen that in few centres these policies have been conceptualized but not materialized. Organizational initiation is much more effective since the best practices are the same for all hospitals and this helps in standardizing the policies. There are organizations which work in promoting IPAC through education, standards, and advocacy and consumer awareness. Examples of organizations working in this field are IPAC Canada, Centers for Disease Control and Prevention (CDC) USA, Infection Prevention Society UK, Asia Pacific Society of Infection Control (APSIC), World Health Organization (WHO). In Nepal organizational initiation to address the issues of IPAC has been recently taken by Healthy Life Foundation Nepal (HELF Nepal) which is an organization with the mission to inform, promote and implement best practices of IPAC to prevent HAIs in the patients as well as the healthcare workers in all healthcare settings in Nepal.

In Nepal awareness on IPAC in Pathology Laboratories can be brought about by initiating trainings, surveillance, regular CMEs and demonstration of techniques to the Lab personnel. Administration will have to be involved in initiating the program and maintaining it with administrative resources and financial support. Before it is too late we have to address the issues of HAIs, AROs and safety in our laboratories.

Dr. Reetu Baral, MD Associate Professor, Department of Pathology Patan Academy of Health Sciences 